\title{
Docencia universitaria semipresencial. Experiencia en el uso de la plataforma virtual SWAD
}

\author{
Manuel López-Morales, Matilde Celma-Vicente, M. Dolores Cano-Caballero Gálvez, Aurora Quero-Rufián, \\ M. Ascensión Rodríguez-López
}

Introducción. La innovación en la metodología docente universitaria es una exigencia del nuevo Espacio Europeo de Educación Superior. La utilización de las tecnologías de la información y la comunicación es una realidad de la sociedad actual, y especialmente del alumnado universitario.

Materiales y métodos. Este artículo muestra la experiencia en el uso de la plataforma virtual de apoyo a la docencia, en la asignatura de Salud y Medio Ambiente en la Universidad de Granada.

Resultados y conclusiones. La opinión de los alumnos es muy favorable hacia su uso. Su nivel de satisfacción es muy alto, aunque les supone una dedicación y esfuerzo mayores a los esperados. La plataforma virtual ofrece unas posibilidades académicas que permiten el acercamiento y adaptación a los nuevos perfiles de alumnado. Para el profesorado supone un cambio en sus principales actividades: la preparación y diseño de contenidos, las tutorías virtuales y la gestión de foros.

Palabras clave. Educación B-learning. Educación basada en competencias. Educación Grado de Enfermería. Técnicas educativas.

Semi-presential university teaching. An experience in the use of SWAD virtual platform

Introduction. The new European Space of higher education demands innovation in the university teaching methodology. The use of the new information and communication technologies is a reality in our current society, especially among university students. The use of virtual platforms for teaching is more developed in post graduate lifelong learning than in university education.

Materials and methods. This articles narrates an experience in the use of SWAD virtual platform, teaching aid, in the subject course Health and Environment, in the University of Granada.

Results and conclusions. The students have a positive opinion about the use of this platform. Their satisfaction level is very high, although it means more dedication and effort than expected. This virtual platform offers academic possibilities that allows us to get close and adapt to the new student profiles. To professors, it means a change in their main activities: preparing and designing contents, virtual tutorials, and forums managing.

Key words. Competency-based education. Distance education. Educational techniques. Graduate Nursing Education.

\section{Introducción}

El Espacio Europeo de Educación Superior (EEES) plantea un modelo diferente para la formación universitaria, en el que se hace énfasis en la enseñanza por competencias centrada en el estudiante, siendo éste el responsable de su propio aprendizaje, y en la que destaca el papel del profesorado como orientador de dicho proceso. Se busca que el alumnado adquiera un compromiso activo sobre el qué y el cómo de lo que aprende; esto supone un cambio en la modalidad de la enseñanza y la utilización de nue- vas metodologías docentes, más adaptadas a las necesidades actuales [1,2]. El cambio de modelo de enseñanza pretende que las competencias que se adquieran vayan más allá de la mera especialización técnica, para incluir, además, dimensiones relacionales y sociopolíticas. Es decir, el comportamiento técnico y metodológico ha de completarse con un comportamiento personal y social [3-6].

La formación de las futuras enfermeras en el ámbito europeo se está configurando dentro de este marco. Todo docente sabe las dificultades para alcanzar estas competencias [7] debido a la masifica-
Escuela Universitaria de Enfermería Virgen de las Nieves. Universidad de Granada. Granada, España.

Correspondencia:

Prof. Manuel López Morales. Escuela Universitaria de Enfermería Virgen de las Nieves. Universidad de Granada. Avda. Fuerzas Armadas, 2. E-18014 Granada. Fax: +34958020132.

E-mail: manuel.lopez.morales.sspa@ juntadeandalucia.es 
ción de aulas, a una elevada carga docente y a una cultura que ha enfatizado la enseñanza basada en clases magistrales, más centrada en el saber que en el saber hacer $[8,9]$.

Este nuevo reto de la enseñanza universitaria es a menudo rechazado en la medida en que pone en cuestión creencias fuertemente arraigadas entre el profesorado, como la vinculación exclusivista a las asignaturas, una infraestructura enfocada fundamentalmente a las clases presenciales, reticencias a ser evaluado por el alumnado y la falta de habilidades en el manejo de nuevas metodologías de enseñanza $[1,8]$.

Desde los años setenta, numerosos autores han mostrado sus diferentes experiencias docentes sobre la modalidad de enseñanza no presencial, resaltando la importancia del aspecto autodidacta del estudiante, la utilización de materiales y contenidos bien estructurados, a los que el alumnado pueda recurrir de manera puntual [10], y la importancia de la interactividad e intercambio bidireccional entre el alumnado [11,12]. Otros autores subrayan la accesibilidad y eficiencia de este tipo de enseñanza al ahorrar desplazamientos e infraestructuras físicas que permiten atender a alumnos separados geográficamente [12,13]. Otros comparan el método con una forma industrializada de impartir la educación [11].

Las universidades españolas han optado por el uso de las tecnologías de la información y la comunicación $[14,15]$. Así, la Universidad de Granada (UGR), ya en el año 1999 [16,17], presentó una plataforma web de apoyo y gestión docente -cuyo nombre es 'Sistema Web de Apoyo a la Docencia' (SWAD), disponible en: https://swad.ugr.es/- integrada desde el 2008 en el Centro de Enseñanzas Virtuales de la UGR. Este recurso permite el seguimiento de las asignaturas mediante la modalidad blended learning (B-learning), en la que el alumnado lleva a cabo un proceso de aprendizaje autónomo bajo la supervisión del profesorado [18]. Para ello, dispone de materiales didácticos seleccionados por el docente y accesibles a través de la propia plataforma [19].

La modalidad de enseñanza B-learning tiene como objetivo alcanzar no sólo las competencias específicas de la asignatura, sino también las competencias transversales, cuya relevancia queda señalada en los planes de estudios del Grado de Enfermería, en el marco del EEES [1]. Destacamos, entre otras, las habilidades de gestión de la información, resolución de problemas, habilidad para trabajar de forma autónoma, diseño y gestión de proyectos, iniciativa y espíritu emprendedor.

Actualmente, la mayoría de los estudiantes dispone de un ordenador con conexión a Internet, por lo que esta herramienta es una opción válida para integrar las posibilidades de formación que ofrece este recurso.

\section{Objetivo general}

Conocer la utilidad de la modalidad B-learning para la docencia universitaria, en la asignatura 'Salud y Medio Ambiente'. Esto permitirá mejorar sus puntos débiles y potenciar los fuertes para poder extender el método a otras asignaturas del Grado de Enfermería.

\section{Objetivos específicos}

1. Conocer la valoración, cualitativa y cuantitativa, que hace nuestro alumnado de la metodología semipresencial para la docencia universitaria.

2. Analizar los puntos fuertes y débiles encontrados por el alumnado en el uso de la plataforma virtual SWAD.

3. Recoger las propuestas de mejora en el uso de la plataforma realizadas por el alumnado.

4. Conocer los puntos fuertes y débiles en opinión de los profesores.

\section{Materiales y métodos}

Trabajo de implementación y mejora tecnológica realizado en la Escuela Universitaria de Enfermería Virgen de las Nieves durante el curso 2009-2010, que constó de dos fases: implantación y evaluación del método.

\section{Descripción del método implementado}

Para esta asignatura se definieron las siguientes competencias específicas:

- Conocer la relación entre los distintos factores de riesgo medioambientales y la salud de las personas.

- Capacidad de identificar la presencia de factores de riesgo medioambientales para la salud humana.

- Capacidad de planificar, desarrollar y evaluar la intervención comunitaria en salud medioambiental.

La distribución de las actividades de la asignatura de Salud y Medio Ambiente se organiza de la siguiente manera:

- Cuenta inicialmente con dos sesiones presenciales: a) Se describen las directrices del trabajo a realizar y se proyecta un vídeo sobre las cuestiones básicas de la asignatura, con el fin de estimular el debate y despertar la motivación. 
b) Participación de dos profesoras invitadas, expertas en materias afines a la asignatura (salud alimentaria y gestión de residuos sanitarios).

- Durante las semanas siguientes los estudiantes leen documentos de distinto tipo, colgados en la plataforma SWAD: artículos de prensa, vídeos, informes de diversas instituciones, guías de actuación medioambiental, legislación sobre el medio ambiente, así como las presentaciones, como las que se proyectarían en una clase magistral, donde se resumen los conceptos fundamentales de cada tema.

- Cada uno de los once temas tiene asignada una actividad individual que el alumnado debe subir a la plataforma en un plazo aproximado de una semana. Son pequeños ensayos (un par de páginas) que los alumnos deben desarrollar sobre el tema en cuestión, como por ejemplo: 'describir los posibles efectos en la salud humana del modelo intensivo de producción agrícola', 'discutir la dificultad para establecer estándares en los riesgos ambientales', 'explicar el tratamiento biológico de las aguas residuales', etc.

- Para cada tema se abre un foro en la plataforma SWAD. En él, se exponen las distintas opiniones de los alumnos a partir de una pregunta que realiza el profesor (p. ej.: ¿somos los humanos una plaga?, ¿son seguros los campos electromagnéticos de nuestros teléfonos móviles?, ¿son inocuos los aditivos alimentarios?). Los debates online estimulan la argumentación y racionalización de concepciones previas sobre la salud medioambiental. El profesor estimula y reconduce los debates para hacer florecer aquellos conceptos clave de cada tema.

- Paralelamente, los alumnos, distribuidos por parejas, elaboran un trabajo monográfico sobre un tema de su interés, entre un listado previo que se les oferta el primer día de clase, especificando los criterios de evaluación que se aplicarán (Tabla I).

- Las tutorías, tanto presenciales como online, apoyan este trabajo.

- La parte final de la asignatura es presencial para realizar la presentación pública y defensa de los trabajos monográficos entre los compañeros. Éstos participan en la evaluación de la calidad de las presentaciones. La heteroevaluación supone el 25\% de la calificación del trabajo. El profesor aporta el otro $75 \%$.

- El sistema de mensajería de la plataforma también es muy utilizado, tanto por los alumnos para concertar/realizar tutorías virtuales, como por el profesorado para mandar instrucciones, advertir de plazos de entrega, sugerir modificaciones en los trabajos, etc.
Tabla I. Criterios para la evaluación del trabajo bibliográfico.

Identificación completa e inequívoca

Estructura de los epígrafes apropiada al contenido

Consistencia y coherencia del contenido

Relevancia de la información aportada

Información contrastada y actualizada

Definición de factores de riesgo

Identificación de problemas de salud asociados

Propuesta de intervención

Conclusiones y reflexiones significativas

Referencias bibliográficas completas (normas de Vancouver)

Claridad expositiva

- La calificación final del alumnado se determina con las siguientes proporciones: actividades individuales, $30 \%$; participación en los foros, $10 \%$; trabajo bibliográfico grupal, $40 \%$, y participación en los debates y evaluación de los trabajos, $20 \%$. La calificación media de los alumnos, en el curso 2009-2010, fue de 8,12 sobre 10 .

\section{Evaluación mediante cuestionarios}

Una vez desarrollada la asignatura, se ofreció al alumnado una encuesta anónima y voluntaria de evaluación (Tabla II), cuya respuesta analizamos en el epígrafe de resultados. De los 39 matriculados, respondieron 33 sujetos.

Para el objetivo 1 se hicieron preguntas dirigidas a conocer el grado de satisfacción con el método, mediante una escala tipo Likert. Para los objetivos 2 y 3 se formularon preguntas abiertas dirigidas a conocer los puntos fuertes y débiles en opinión del alumno. Para el objetivo 4 se sometió toda la información obtenida a debate entre el profesorado en un grupo de discusión.

\section{Resultados}

\section{Valoración cuantitativa}

Las valoraciones medias (sobre 10) adjudicadas por los alumnos fueron las siguientes: documentación, 
Tabla II. Evaluación de la asignatura por parte del alumnado.

Califique, por favor, de 0 a 10 su grado de acuerdo con las afirmaciones siguientes:

El contenido de la asignatura ha respondido a mis expectativas

La documentación facilitada es apropiada para el desarrollo de los contenidos

La metodología de la asignatura es adecuada

La plataforma virtual ha facilitado mi aprendizaje

La tutorización recibida ha sido apropiada

Relate, por favor, los aspectos cuestionados seguidamente:

Lo que más me ha gustado de la asignatura ha sido:

Dificultades/problemas encontrados en la asignatura:

Propuestas de mejora de la asignatura:
La densidad de la documentación la resaltan como una dificultad. Documentos muy numerosos, extensos y dispersos, según sus apreciaciones: 'documentación muy extensa, 'alguna documentación no concordaba con la actividad relacionada, 'diversidad de temas tratados...

También hay alguna observación sobre problemas técnicos para colgar las actividades en la plataforma, bien por lo ajustado de los plazos de entrega, bien por el formato aceptado por ella: 'la plataforma SWAD a veces no me dejaba insertar los documentos', 'periodos de entrega demasiado estrictos', 'problemas con el docx/doc'...

\section{Propuestas de mejora}

Las propuestas de los alumnos se dirigen sobre todo a la disminución de la carga de trabajo: 'es una asignatura que conlleva más horas de trabajo que los créditos reconocidos', 'más tiempo para las actividades,' 'menos carga de trabajo,' 'más tiempo para la asignatura, 'reducir el número de artículos', 'que la información aportada sea más sencilla'...

No falta quien echa de menos metodologías más clásicas: 'más clases presenciales para hacer explicaciones, 'dar los apuntes por escrito', 'más participación en el foro del profesor'...

Las preguntas abiertas del cuestionario se sometieron a un análisis de contenido que sintetizamos a continuación:

\section{Sobre las fortalezas del método}

Se destaca sobre todo la ventaja del trabajo a través de la plataforma, que permite adaptar los horarios, gestionar el propio tiempo y trabajar a distancia: 'metodología que permite adaptar el horario de trabajo,' 'poder trabajar desde casa, 'que no sea presencial...

También se valoran muy positivamente los contenidos de la asignatura y la documentación facilitada: 'he aprendido mucho sobre el tema ya que he leído muchos documentos relacionados', 'he aprendido bastantes cosas interesantes sobre la asignatura,' 'abordaje de temas de interés para la vida actual', 'la variedad de temas ayuda a aprender muchas cosas,' 'los enlaces permiten resolver muchas dudas'...

\section{Puntos débiles}

Se quejaban de la intensidad del trabajo, que exige mucha dedicación de tiempo y esfuerzo: 'mucho trabajo para tan poco tiempo', 'hay actividades que cuesta mucho realizarlas para lo poco que valen,' 'la asignatura necesita mucho tiempo de dedicación, 'actividades muy seguidas y algunas de ellas muy densas...

\section{Opinión del profesorado}

El profesorado participante se reunió en un grupo de discusión para debatir sobre su experiencia con la plataforma SWAD. Todos desarrollan sus asignaturas con apoyo, en mayor o menor grado, de la plataforma virtual. De ese grupo de discusión se extraen las siguientes conclusiones:

- Se identifica la necesidad de una formación previa para el uso de la plataforma, tanto para el alumnado como para el profesorado. Las dificultades en su manejo, al principio de su uso, resta efectividad.

- La plataforma virtual incrementa el esfuerzo del profesorado en la planificación, compilación y gestión de materiales. También en la homogenización y estandarización del trabajo del alumnado, definiendo las normas, formatos, criterios de evaluación y plazos de entrega de las actividades. Debe extremarse la claridad y exactitud de las instrucciones.

- Aunque parezca contradictorio, se establece una relación más personalizada entre profesor/a y alumno/a, y se incrementan las sesiones de tutorización, por supuesto las tutorías online, pero también las presenciales. Esto no es así siempre. En otros casos puede perderse el contacto perso- 
nal, lo que dificulta la evaluación del trabajo de los alumnos. Vía online no pueden valorarse las actitudes. Esto ejemplifica la potencialidad de esta metodología en la diversificación y atención personalizada a cada tipo de alumno. Por otra parte, suscitan algunas dudas sobre la autoría de los trabajos, pues se facilita la suplantación de identidad.

- El tiempo dedicado a los foros es cuantioso y la evaluación de éstos, compleja.

- La coordinación entre el profesorado es esencial. La facilidad para colgar materiales puede inducir a un sobredimensionamiento de la documentación, que puede ser común entre asignaturas, en consonancia con la transversalidad de algunas competencias buscadas. Planificar conjuntamente y compartir de forma explícita materiales didácticos orienta mejor a los estudiantes y satisface los objetos del EEES.

- La documentación de los despachos se reduce. El formato electrónico permite guardar grandes cantidades de información en reducidos espacios.

\section{Discusión}

La docencia semipresencial de esta asignatura es bien valorada por los alumnos y la plataforma virtual constituye una buena herramienta para su desarrollo, ya que les permite compaginar los estudios con otras actividades y organizar el tiempo de una manera flexible. Esto coincide con otros estudios donde se muestra que la plataforma virtual permite el acercamiento y adaptación a los nuevos perfiles del alumnado [20-22].

Así mismo se ha mostrado idónea para alcanzar otras competencias transversales, como el uso de nuevas tecnologías y el trabajo en equipo $[7,19]$. Atendiendo a las altas calificaciones obtenidas por los estudiantes, las competencias específicas también se ven satisfechas. Una investigación a más largo plazo compararía el impacto en la formación competencial efectiva respecto a otras modalidades de enseñanza. Un reto para próximas investigaciones.

Los nuevos formatos docentes basados en el uso de las nuevas tecnologías de la información y la comunicación contribuyen a la adaptación a los nuevos modelos académicos derivados del EEES [1]. Esto supone un cambio de cultura tanto del alumnado como de los profesores, que requiere todavía más esfuerzo, como otros autores confirman $[7,19]$.

En este sentido, desde el punto de vista del profesorado, cabe destacar la modificación del rol profesional con la metodología semipresencial. Ya no se trata de dictar conceptos para que sean recogi- dos por el alumnado (con diferente capacidad de cribado). Se trata de la construcción real de conocimiento por parte del alumnado ('aprender a aprender'). Pero esto, contrariamente a lo que podría parecer, inicialmente, incrementa de manera notable la carga de trabajo del profesor, por lo que exige un esfuerzo de regulación [23].

La clave del papel del profesorado reside, por una parte, en la selección de contenidos y su organización temática, y por otra, en la revisión de los trabajos de los alumnos y su debate conjunto, utilizando los foros y las tutorías virtuales. La evaluación es una función docente que se hace más difícil - pero sobre todo se modifica - con la metodología semipresencial.

Es necesaria la coordinación entre las asignaturas para evaluar la carga de trabajo no presencial del alumno y ajustarla a los créditos ECTS que conforman cada asignatura, una reclamación de los alumnos que otros autores también advierten [24]

La plataforma SWAD ha mostrado ser una buena herramienta para la formación académica, que hay que saber manejar y sacarle provecho. El profesorado no puede permanecer al margen del desarrollo tecnológico, pues no sólo los contenidos, sino también el modo de desarrollarlos, son claves para la calidad de la enseñanza.

\section{Bibliografía}

1. Agencia Nacional de Evaluación de la Calidad y Acreditación (ANECA). Libro Blanco de la Titulación de Enfermería. 2005. URL: http://www.aneca.es/media/150360/ libroblanco_jun05_ enfermeria.pdf. [05.02.2008].

2. Celma M. Cultura organizacional y desarrollo profesional de las enfermeras [tesis doctoral]. Granada: Universidad de Granada; 2008.

3. Le Boterf G. De la compétence a la navigation professionnelle. Paris: Editions d'Organisations; 1996.

4. Gilbert P, Parlier M. La compétence: du 'mot-valise' au concept opératoire. Actualité de la Formation Permanente 1992; 116: 14-8.

5. Bunk GP. La transmisión de las competencias de la formación y perfeccionamiento profesionales. Revista Europea de Formación Profesional 1994; 1: 8-14.

6. Fuller A. Purpose, value and competence: contextualising competence based assessment in the civil aviation sector. Br J Educ Work 1995; 2: 60-75.

7. Bolaños JE, Pérez J. Como fomentar las competencias transversales en los estudios de ciencias de la salud: una propuesta de actividades. Educ Med 2005; 8: 216-25.

8. Guillamet A, Celma M, González P, Cano-Caballero MD, Pérez F. Aprendizaje basado en problemas. Estrategias de implantación. Revista Rol de Enfermería 2009; 32: 124-8.

9. Celma M, Montalbán S, López M, Corral C, Guillamet A, De la Rosa A. Proyecto de mejora de la enseñanza teóricopráctica en la asignatura médico-quirúrgica. Revista Metas de Enfermería 2010; 13: 67-74.

10. Holmberg B. Distance education -a survey and bibliography. London: Kogan; 1977.

11. Keegan D. Foundations of distance education. London: Routledge; 1990. 
12. Kaye A, Rumble G. An analysis of distance teaching systems. Milton Keynes, UK: Open University; 1979.

13. Martín-Ibáñez, R. Introducción a las ciencias de la educación. Madrid: UNED; 1986.

14. Castellanos NN, Cirre CM, González J, Lacasa D, MartínezSantaolalla MG. Enseñanza virtual para el apoyo a la docencia de la Universidad de Almería. Jornadas sobre la Influencia de las NTICs en el Campo Docente. Almería: Universidad de Almería; 2005.

15. Carrasco A, Gracia E, De la Iglesia C. Las TIC en la construcción del Espacio Europeo de la Educación. Dos experiencias docentes en teoría económica. Revista Iberoamericana de Educación 2005; 36: 1-15.

16. Cañas A, Díaz AF, Rodríguez M, Bernier JL., Prieto A. Development and evaluation of web-based tool to support university education and administration. Information society and education: monitoring a revolution. Serie Sociedad de la Educación 2002; 9: 473-7.

17. Cordón O, Anaya, K. Jornadas Thales. Granada: CEVUG. UGR; 2004.

18. Bartolomé A. Blended learning. Conceptos básicos. Monográfico Blended Learning. Pixel-Bit, Revista de Medios y Educación 2004; 23: 7-20.

19. Cañas A, Calandria DJ, Ortigosa EM, Ros E, Díaz AF. SWAD: web system for education support. In FernándezManjón B, Sánchez-Pérez JM, Gómez-Pulido JA, VegaRodríguez MA, Bravo-Rodríguez J, eds. Computers and education: e-learning -from theory to practice. New York: Springer; 2007. p. 133-42.

20. Cano-Caballero MD, Celma M, Pérez F, Jerez JM, Garach JI. Usos y utilidades de una plataforma virtual en la formación universitaria de enfermería. V Jornadas de Profesorado de
Centros Universitarios de Enfermería. Herramientas de trabajo en la evaluación por competencias: LAS tecnologías de la información y la comunicación (TICs y la rúbrica). Murcia, 2011.

21. Rodríguez-López MA, Guillamet A, Heirele C, LópezMorales M, González I, Quero A. Percepción del profesorado sobre el uso de una plataforma virtual par ala formación universitaria de enfermería. V Jornadas de Profesorado de Centros Universitarios de Enfermería. Herramientas de trabajo en la evaluación por competencias: las tecnologías de la información y la comunicación (TICs y la rúbrica). Murcia, 2011.

22. Quero A, Guillamet A, Pérez F, González P, Garach J, Villanueva C. Valoración cualitativa del alumnado sobre el aprendizaje basado en problemas. XII Encuentro de Investigación en Enfermería y $6{ }^{\circ}$ Coloquio Bianual Joanna Briggs. Madrid: Instituto de Salud Carlos III/Unidad de Coordinación y Desarrollo de la Investigación en Enfermería; 2008. p. 397-8.

23. Valencia MC, Cruces C, González A. Innovación docente en química analítica instrumental. Jornadas de Trabajo sobre Experiencias Piloto de Implantación del Crédito Europeo en las Universidades Andaluzas. Cádiz, 2006. URL: http:// www2.uca.es/orgobierno/rector/jornadas/documentos/085. pdf. [10.10.2010].

24. Azuaga A, Berenguer MC, Blázquez G, Calero F, Extremera J, García AM, et al. Experiencias sobre actividades académicamente dirigidas y actividades transversales. Jornadas de Trabajo sobre Experiencias Piloto de Implantación del Crédito Europeo en las Universidades Andaluzas. Cádiz, 2006. URL: http://www2.uca.es/orgobierno/rector/jornadas/ documentos/163.pdf. [10.10.2010]. 